Evaluating the intensity for the different modes gave a minimum of zero emission at $\theta=0$ for the $T E_{01}$ and $T M_{01}$ modes, and maxima in the range of $30-50^{\circ}$. On the other hand, the $H E_{11}$ mode had a maximum emission at $\theta=0$. The radius of the nanowire directly affected the intensity of the response of each mode, but much more for the $T M_{01}$ and $H E_{11}$ modes than for the $T E_{01}$ mode. Experimentally, polarization measurements will determine the mode type and should be taken close to the top end of the nanowire to avoid interference with the radiation from the bottom end.

The researchers said that the ability to control the properties of laser emission from nanowires may open new possibilities in optoelectronics in conjunction with self-waveguiding and the possibility of nanowire array fabrication.

SIARI SOSA

\section{Simulation of Spin-MOSFET Predicts Strong Dependence of Spin Transport on Magnetic Configuration}

Spin transistors utilizing two ferromagnetic layers as spin injector and spin analyzer, respectively, possess unique output characteristics that are controlled by the relative magnetization configuration of the ferromagnets as well as bias conditions. Together with the ability to utilize the latter as nonvolatile binary data, spin transistors could allow the fabrication of ultrahigh-density nonvolatile memory in integrated circuits (ICs) in which the memory cell consists of a single spin transistor as well as functional spin-transistorgate-based nonvolatile reconfigurable logic. In order to attain high-performance "spintronic" integrated circuits, five requirements need to be fulfilled: (1) a large magnetocurrent ratio for nonvolatile memory and logic functions; (2) high transconductance for high-speed operation; (3) a high amplification capability (voltage, current, and/or power gains) to restore propagating signals between transistors; (4) a small power-delay product and small off current for low power dissipation; and (5) a simple device structure for a high degree of integration and high process yield.

Until now, proposed spin transistor designs have been unable to satisfy all of these requirements. The simultaneous achievement of high transconductance and amplification capability with a large magnetocurrent ratio has been difficult. S. Sugahara and M. Tanaka of the Department of Electrical Engineering at the University of Tokyo, however, have conducted a two-dimensional numerical analysis of their spin-type metal oxide semiconductor field-effect transistor (MOSFET) design, fulfilling all five of these requirements and predicting highamplification capability, a low powerdelay product, a low off current, and extremely large magnetocurrent ratios.

As reported in the March 22 issue of Applied Physics Letters, the designed spin MOSFET consists of a MOS gate structure and half-metallic ferromagnet (HMF) contacts for the source and drain, similar to a Schottky source/drain MOSFET except for the HMF source/drain contacts, which are $\mathrm{HMF} / \mathrm{Si}$ junctions without $p-n$ junctions. The device simulated used a channel length of $30 \mathrm{~nm}$, a Si layer thickness of $10 \mathrm{~nm}$, and an oxide layer thickness of 2-3 $\mathrm{nm}$. The design can be applied not only to $n$ - and $p$-channel devices, but also to accumulation- and inversion-type channel devices. Promising HMF materials include Heusler alloys and oxides such as $\mathrm{CrO}_{2}$ and $\mathrm{Fe}_{2} \mathrm{O}_{3}$. Of even more interest are halfmetallic compounds exhibiting the zincblende crystal structure, such as CrAs, $\mathrm{MnAs}$, and $\mathrm{CrSb}$, since their predicted high Curie temperature and large bandgap of the insulating spin band are most useful as HMF source/drain materials, said the researchers.

Sugahara and Tanaka found that their simulations predict that the HMF source not only functions as a contact for blocking an off current, but also acts as a spin injector of up-spin electrons from the HMF source to the channel. The HMF drain selectively extracts the up-spin electrons from the channel when the magnetizations of the HMF source and drain are parallel. The scientists expect an extremely large magnetocurrent ratio due to the high spinselectivity of the HMF source/drain when these spin-filter effects are combined. The output characteristics were calculated using the Tsu-Esaki formula with a twodimensional transmission probability calculation and gave magnetocurrent ratios of $>1000 \%$ at $<1.0 \mathrm{~V}$ drain/source bias $V_{\mathrm{DS}}$ and even larger ratios at smaller $V_{\mathrm{DS}}$. Other calculated device parameters such as transconductance were similar to those that have been obtained for MOSFETS with gate lengths of $<100 \mathrm{~nm}$.

Alfred A. ZiNN

\section{Effects of Chirality on Goos-Hänchen Phase Shifts at Interfaces in Optical Media Explored}

A light beam that is totally reflected at the interface between two different media always undergoes a phase change. Light waves with different incident wave vectors are subject to different magnitudes of this phase change. As a result of this inter- ference among the plane-wave components with different incident wave vectors, the weight center of the reflected beam is displaced laterally with respect to the weight center of the incident beam, a phenomenon known as the Goos-Hänchen shift. This event includes both a phase shift that can either reinforce or oppose the incident wave vector and a lateral beam shift that is either in the same or opposite direction as the horizontal component of the energy flux of the incident beam. Recently, D.-K. Qing and G. Chen from the Department of Mechanical Engineering at the Massachusetts Institute of Technology investigated Goos-Hänchen shifts at the interfaces between optical media of different "handedness," or chirality.

The Goos-Hänchen shifts are well known for total reflection of light propagating in conventional dielectric materials. Such media are known as "righthanded" materials, since the wave vector, electric-field vector, and magnetic vector of light beams traveling in them form a right-handed system. However, in media that have negative permeability and negative permittivity, these vectors form a lefthanded system, and thus the medium is called "left-handed." Although left-handed materials have attracted recent interest for new devices such as novel optical waveguides, superlenses, and microcavities, no previous study exists in which the light propagating through them was incident from the left-handed medium.

As reported in the April 15 issue of Optics Letters, Qing and Chen have determined mathematically the Goos-Hänchen phase changes and lateral beam shifts for beams propagating in both left-handed and right-handed media, reflected at interfaces between both same-handed and different-handed materials. This gives four possible situations. In a concise diagram, the researchers elucidate the differences between Goos-Hänchen shifts for each possible system. When the two materials are both of the same handedness (whether left- or right-handed), the Goos-Hänchen phase shift opposes the phase change resulting from propagation through the incident medium. This change is positive in right-handed systems and negative in left-handed ones. The Goos-Hänchen lateral shift between two same-handed systems is in the same direction as the horizontal component of the incident energy flux. Alternatively, when the two media are of opposite handedness, the Goos-Hänchen phase shift reinforces the phase variation of the incident beam, while the lateral shift is opposite the horizontal component of the incident energy flux. These observations are true for inter- 
faces between different-handed materials regardless of the medium in which the beams are propagating.

\section{ANDY FRANCIS}

\section{Layered ZnDS Nanocomposites Formed with Assistance of PLA}

Researchers have paid considerable attention recently to the family of layered metal hydroxide materials, such as layered double hydroxides (LDHs), hydroxide double salts (HDSs), and single-metal hydroxides because of their pronounced anion exchange capacity. N. Koshizaki and colleagues from Nanoarchitectonics Research Center, Japan, formed layered zinc hydroxide/dodecyl sulfate (ZnDS) nanocomposites in a special solution environment created by pulsed laser ablation (PLA) of a zinc plate in an aqueous solution of sodium dodecyl sulfate (SDS). As reported in the February 24 issue of Chemistry of Materials, the researchers made the preparation of ZnDS a simple process, directly triggered by metal species without any chemical modifications.

Other methods for the synthesis of layered composites-for example, zinc hydroxide salts (LHS-Zn)-are coprecipi- tation or organo-derivatization reaction methods, but such LHS-Zn composites are generally poorly crystallized and exhibit turbostratic disorder.

In contrast, the researchers obtained a ZnDS composite platelet that presents highly ordered single-crystalline layered structures with well-defined octagonal shapes.

The scientists conducted PLA experiments in deionized water with dilute $(0.001 \mathrm{M})$ and more concentrated $(0.1 \mathrm{M})$ SDS solutions. X-ray diffraction of the resultant ZnDS composite showed the formation of layered structures: ZnDS products with long-range order and with no turbostratic faults revealed in products from either SDS solution and some stacking disorder indicated in products from the 0.1 M SDS solution. Products by PLA of $\mathrm{Zn}$ in deionized water revealed typical reflections of wurtzite $\mathrm{ZnO}$, also confirmed by transmission electron microscopy (TEM) observation.

TEM images of products in $0.001 \mathrm{M}$ or $0.01 \mathrm{M}$ SDS solutions revealed numerous lamellar thin platelets with irregular octagonal shapes and an average diameter of $1.5 \mu \mathrm{m}$ preferentially lying on the grid. Electron diffraction patterns showed that the octagonal platelets were monocrystals lying naturally on the plane with clear hexagonal crystal symmetry.

In the composite formation processes, the researchers produced charged inorganic zinc hydroxide species step-by-step by the strong reaction between the ablated $\mathrm{Zn}$ species and the water molecules. The preferred coordination of hydrophilic head groups with zinc coordination sites prevents further reaction from forming $\mathrm{ZnO}$ nanoparticles.

The researchers said their simple method of forming layered $\mathrm{ZnDS}$ nanocomposites enables the development of new types of hybrid composites by using other applicable metal targets and surfactants.

EKATERINA A. LitVINOVA

\section{Carbon-Nanotube Formation Observed In Situ}

Due to their magnetic, electronic, and mechanical properties, carbon nanotubes (CNTs) are excellent candidates for a variety of emerging nanotechnology applications. Exploitation of any of these properties requires either the production or the

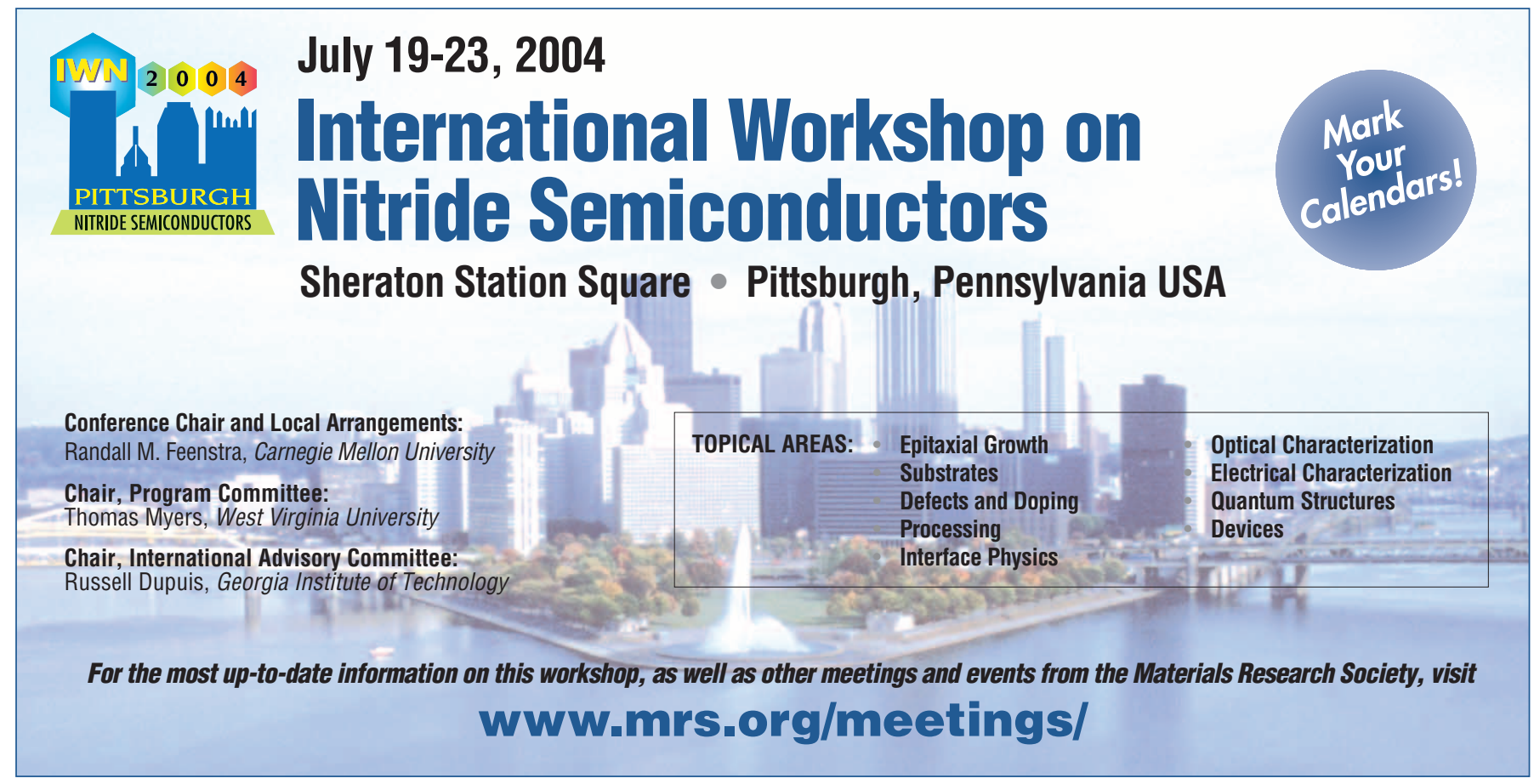

Exhibitors... Availability is limited so reserve your booth space today! Contact MRS at 724-779-8312 